\title{
The geography of parasite local adaptation to host communities
}

\author{
Emily Bellis ${ }^{1}$, Chloee McLaughlin ${ }^{2}$, Claude DePamphilis ${ }^{2}$, and Jesse Lasky² \\ ${ }^{1}$ Arkansas State University \\ ${ }^{2}$ The Pennsylvania State University
}

February 2, 2021

\begin{abstract}
Fitness responses to environment can shape species distributions, though opposing eco-evolutionary processes can obscure environmental effects. For example, host specificity influences parasite dynamics, but is unclear how adaptation of parasites to local host communities may scale up to continental distributions. Here, we develop a macroecological framework to determine how host community structure affects the distribution of specialist and generalist populations of Striga hermonthica, an African parasitic plant of cereal crops. Combining data from global crop production and parasite experimental trials, we find that parasites perform best on the host species that is most common in their location of origin. Moreover, niche model contrasts predict parasite specialization on two hosts that evolved alongside Striga during domestication (pearl millet and sorghum), indicating that specialist parasites may be most likely to occur where host niches differ most in multivariate environmental space. Our study demonstrates that patterns of parasite local adaptation to host communities can emerge at continental scales and that differential environmental tolerances of hosts indirectly shape the distribution of specialist and generalist parasites. By predicting spatial dynamics of parasite specialization versus generalization directly from environmental data, our approach may help inform current and future management of pests and disease.
\end{abstract}

Title: The geography of parasite local adaptation to host communities

Authors: Emily S. Bellis ${ }^{*}$, Chloee M. McLaughlin ${ }^{2,3 *}$, Claude W. dePamphilis ${ }^{3}$, and Jesse R. Lasky ${ }^{3}$

$*^{*}$ co-first authors

\section{Author Affiliations:}

${ }^{1}$ Arkansas Biosciences Institute and Department of Computer Science, Arkansas State University, State University, AR 72467

${ }^{2}$ Intercollege Graduate Program in Plant Biology, The Pennsylvania State University, University Park, PA 16802

${ }^{3}$ Department of Biology, Pennsylvania State University, University Park, PA 16802

\section{Author Contact:}

ESB: ebellis@astate.edu; CMM: cmc7333@psu.edu; CWD: cwd3@psu.edu; JRL: jr135@psu.edu

Running Title: Macroecology of host specialization

Type of Article: Letters

Number of Words: Abstract: 150; Main text: 4,891

Number of References:

Number of Figures, Tables, and Text Boxes: 5 Figures, 0 Tables, 0 text boxes 


\title{
Corresponding Author :
}

Emily S. Bellis, Arkansas Biosciences Institute, P.O. Box 639, State University, AR 72467

Email: ebellis@astate.edu

Phone: 870-680-3979

Fax: 870-972-2026

\section{Statement of Authorship:}

ESB, CWD, and JRL conceived of the study. ESB designed geographic and environmental analyses. CMM developed linear mixed models. ESB, CMM, and JRL wrote the manuscript with input from CWD.

\section{Data Accessibility:}

No new data were generated. Code needed to reproduce results of the manuscript are available at https://github.com/em-bellis/StrigaMacroecologyMS

\begin{abstract}
:
Fitness responses to environment can shape species distributions, though opposing eco-evolutionary processes can obscure environmental effects. For example, host specificity influences parasite dynamics, but is unclear how adaptation of parasites to local host communities may scale up to continental distributions. Here, we develop a macroecological framework to determine how host community structure affects the distribution of specialist and generalist populations of Striga hermonthica, an African parasitic plant of cereal crops. Combining data from global crop production and parasite experimental trials, we find that parasites perform best on the host species that is most common in their location of origin. Moreover, niche model contrasts predict parasite specialization on two hosts that evolved alongside Striga during domestication (pearl millet and sorghum), indicating that specialist parasites may be most likely to occur where host niches differ most in multivariate environmental space. Our study demonstrates that patterns of parasite local adaptation to host communities can emerge at continental scales and that differential environmental tolerances of hosts indirectly shape the distribution of specialist and generalist parasites. By predicting spatial dynamics of parasite specialization versus generalization directly from environmental data, our approach may help inform current and future management of pests and disease.
\end{abstract}

\section{Introduction:}

Two central questions in biology are what maintains diversity in environmental responses within species and what determines the distribution of organisms across environmental gradients? These two questions are intimately related but often studied separately. A common hypothesis is that adaptation by populations to different local environments is a major source of diversity in environmental responses within species (Clausen et al. 1940, Kawecki and Ebert 2004). Local adaptation is driven by genetic variation in fitness responses to the environment ("environmental preferences") and thus local adaptation may shape abundance patterns across environments. It is often hypothesized that a species' relative abundance in different habitats reflects environmental preferences, but there are many empirical studies where additional processes such as dispersal (Pulliam 1988) obscure or counteract environmental effects.

Local adaptation is defined as a genotype-by-environment interaction for fitness such that home genotypes have higher fitness than foreign genotypes (Kawecki and Ebert 2004). Local adaptation is common but not ubiquitous (Leimu and Fischer 2008, Hereford 2009) and often difficult to detect in host-parasite systems because parasites may be better adapted to host populations from the recent past than to contemporary host populations (Koskella 2014). The scale of environmental heterogeneity relative to gene flow is one factor determining whether populations adapt to local conditions, evolve generalist strategies (Slatkin 1973, Penczykowski et al. 2016), or specialize on a single environment (Brown and Pavlovic 1992). For example, local adaptation is favored when the size of habitat patches is larger than the characteristic scale of gene 
flow, while smaller habitat patches may favor adaptation to the average of environments encountered, due to the unpredictability of environments inhabited by offspring (Slatkin 1973). This pattern is readily apparent from empirical studies of host-parasite metapopulations, where the scale of parasite adaptation to local host genotypes depends strongly on habitat configuration and relative scales of gene flow (Thrall and Burdon 1997, Thrall et al. 2002, Laine 2005, Koskella et al. 2011, Tack et al. 2014). However, it is unclear how local adaptation in metapopulations scales up to determine species distribution and abundance (Peterson et al. 2019).

A simple hypothesis about distributions is that population size is greatest where environmental conditions are most favorable (Brown 1984). Yet organisms are often excluded from ideal environments by negative biotic interactions such as competition or predation (Buckley and Roughgarden 2005). Organisms can also persistently occur in poor environments due to immigration (Pulliam 1988). Similarly, high extinction rates of parasite populations followed by recolonization, or high adaptability of hosts, can also limit parasite adaptation to their local hosts (Kaltz and Shykoff 1998). As a result, recent studies have questioned the assumption underlying many macroecological studies that patterns of environmental distribution reflect individual fitness responses to environment (Osorio-Olvera et al. 2019, Holt 2020). Relatedly, parasite fitness may not always be highest in environments where they are most abundant, though several studies suggest parasite abundance is greater on preferred hosts (Krasnov et al. 2004, Poulin 2005). Higher abundance may translate to higher frequencies of parasite occurrence across sampled localities (Poulin et al. 2012), but this trend is rarely linked to patterns of local adaptation.

To address macroecological hypotheses of resource specialization, parasite systems are perhaps some of the most promising (Stephens et al. 2016). For example, the growing availability of host-parasite occurrence data has enabled continent-scale investigations of host specificity (Fecchio et al. 2019, Wells et al. 2019), defined as the number or diversity of hosts a parasite can infect (Wells and Clark 2019). In contrast, host specialization (in the Grinnellian sense) refers to variance in species' performance across a range of environments (Futuyma and Moreno 1988, Devictor et al. 2010). Compared to host specificity (Fecchio et al. 2019), macroecological studies of host specialization are scarce, perhaps due to the need for quantitative measures of parasite performance which are generally more difficult to obtain than occurrence data.

Here, we investigate continent-scale patterns of host specialization in the parasitic plant Striga hermonthica. A root hemiparasite of the broomrape family (Orobanchaceae), S. hermonthica is characterized by a complex life cycle. Seeds can remain viable in soils for up to 14 years (Bebawi et al. 1984) until germination, which requires detection of specific hormones (strigolactones) present in host root exudates (Cook et al. 1966). Other host-derived molecules trigger the formation of the haustorium (Cui et al. 2018), a specialized feeding structure used to invade host tissues and form vascular connections. Through these connections, parasitic plants suppress host immune responses (Shahid et al. 2018) and acquire water and nutrients to support their own development, emergence, and reproduction (Clarke et al. 2019). S. hermonthica is widespread across diverse abiotic environments in East and West Africa where it parasitizes grasses including maize, pearl millet, sorghum, sugarcane, and rice and is a major constraint to food security (Rodenburg et al. 2016, Runo and Kuria 2018). Compared to pearl millet (Pennisetum glaucum) and sorghum (Sorghum bicolor), which both have centers of domestication in Africa (Winchell et al. 2017, Burgarella et al. 2018), maize is a relatively recent host and has few natural resistances to Striga (Rich and Ejeta 2008, Timko et al. 2012).

Parasitic plants offer numerous advantages for studying the eco-evolutionary dynamics of parasitism. $S$. hermonthica hosts are characterized by mating systems from highly outcrossing (pearl millet and maize) to predominately selfing (sorghum) and a diversity of abiotic requirements. Unlike many microbial pathogens and endoparasites, parasite generation times are similar to their hosts $(\sim 1$ year) leading to more balanced coevolutionary dynamics. The potential for reciprocal coevolution (rather than asymmetrical adaptation of parasites to hosts often assumed in agricultural settings) is supported by a recent study of sorghum and $S$. hermonthica. Bellis et al. (2020) found evidence that S. hermonthica prevalence imposed spatially-varying selection on sorghum landraces, promoting among-population diversity in sorghum alleles for $S$. hermonthica resistance. $\quad$ Parasitic plants are large and conspicuous, so excellent occurrence data are available from 
natural history collections without a need for dissections or molecular diagnostics. Host specialized as well as generalist populations are known (Parker and Reid 1979). However, the eco-evolutionary determinants of specialization are poorly characterized, despite detailed knowledge at the molecular level on parasitic plant response to different hosts (Honaas et al. 2013, Johnson et al. 2019, Lopez et al. 2019).

We synthesize previous experiments on host specialization, combined with continental scale host and parasite distribution data, to ask three questions: Q1) Does regional abundance of a host crop lead to local specialization by parasites?, Q2) Is host specialization associated with observed patterns of parasite occurrence on different hosts?, and Q3) Do abiotic environmental gradients shape the distribution of specialist and generalist parasites, suggesting that future dynamics of specialization vs. generalization may be predicted by abiotic change?

\section{Materials and Methods:}

\section{Host specialization}

To characterize host specialization, we compiled data from four published empirical studies that tested $S$. hermonthica performance (specifically, "emergence" or the number of above-ground parasites per individual host plant at or near crop maturity) on sorghum, millet, and maize (King and Zummo 1977, Ramaiah 1983, Bebawi et al. 1986, Kim et al. 1994). These data include S. hermonthica populations from 27 locations spanning semiarid regions of East and West Africa between the equator and $14^{\mathrm{O}} \mathrm{N}$ (Fig. 2, 3). $S$. hermonthica populations from empirical studies were classified into regional groupings based on their location of origin coordinates. Populations from localities with longitudinal coordinates less than $0^{\underline{0}}$ were considered "Western", greater than $15^{\mathrm{O}} \mathrm{E}$ were considered "Eastern", and greater than $0^{\circ} \mathrm{W}$ but less than $15^{\mathrm{O}} \mathrm{E}$ were considered "Central". Studies of these populations include both pot experiments and field trials (Table S1) and to our knowledge, represent all studies that have quantified emergence of $S$. hermonthica from geographically distinct populations on multiple genotypes of multiple host species. To account for differences in inoculation pressure and experimental design between studies, for a given $S$. hermonthica population we considered the mean relative emergence on host species $k$ as

$$
\text { Mean Relative } \text { Emergence }_{k}=1 / n \sum_{j=1}^{n} E_{j} / E_{\max }
$$

where $E_{j}$ is the average emergence on host genotype $j, E_{\max }$ is the maximum emergence of the parasite population observed on any host genotype of any host species tested at the same location, and $n$ is the number of genotypes tested of host species $k$. Mean relative emergence ranges from 0 to 1 where, for example, a mean relative emergence of 0.5 on maize would indicate that for a given parasite population, on average half of the individuals emerge on genotypes of maize compared to the maximum emergence in an experiment. Similarly, a mean relative emergence close to 1 on sorghum would indicate that a parasite population specializes on sorghum.

Using these values for mean relative emergence, we then classified $S$. hermonthica populations as generalist or specialist using two complementary approaches. First, we used hierarchical clustering, an unsupervised approach, to identify groups of $S$. hermonthica populations with similar mean relative emergence across the three hosts using the 'hclust' function in R ( R Core Team 2020). Clustering was performed using correlation-based distance, which clusters observations with similar profiles, even if there is large variation in the magnitude of feature values (i.e. mean relative emergence). As the measure of cluster dissimilarity, we used 'complete linkage', which considers the maximum distance between any pair of observations in two clusters. We also considered a continuous measure of host specialization, the Paired Difference Index (PDI), calculated as

$$
P D I=1 /(R-1) \sum_{i=2}^{R}\left(P_{1}-P_{i}\right)
$$


where $\mathbf{P}_{1}$ is the highest link strength, $\mathbf{P}_{1}$ is the link strength with the $i$ th resource, and $R$ is the number of resources (Poisot et al. 2012). For calculations of PDI, mean relative emergence was used as a measure of link strength on each resource (host).

\section{Distribution of host crop communities}

We compiled data on host crop distributions to determine how variation in host ecological communities, i.e.cultivation of specific crops, was associated with parasite specialization (Q1). As a proxy of host abundance, we considered area harvested of the three focal host species: maize, millet, and sorghum from global crop production data (Monfreda et al. 2008). These data are based on integration of national, state, and county level census statistics with global cropland cover derived from satellite imagery and provide estimates of average hectares harvested for each crop per land area of a $5 \times 5$ arcminute grid cell, according to multiple years of data centered on the year 2000 (Monfreda et al. 2008). Although there is a temporal mismatch between crop harvest data and experimental data, the proportion of area harvested of each host crop has remained relatively constant in each country, indicating that the harvest data from 2000 are a good proxy for conditions in earlier years (Fig. S1). We estimated relative abundance of hosts by calculating the fraction of land planted to a particular host relative to maize, millet, and sorghum combined. We also considered productivity (yield per hectare) from the same dataset (Monfreda et al. 2008). Analyses were limited to S. hermonthica prone regions, which we defined as regions within $200 \mathrm{~km}$ of a known occurrence with habitat suitability scores [?] 0.1, according to S. hermonthica environmental niche models (ENMs) constructed using occurrences on all hosts (Bellis et al. 2020).

\section{Parasite occurrence}

To evaluate whether host specialization is associated with parasite occurrence on different hosts (Q2), we calculated the fraction of parasites observed on a particular host, relative to all parasites with an identified host within $50 \mathrm{~km}$ from locations of origin of $S$. hermonthica populations tested in experiments. Starting from an initial set of $S$. hermonthica occurrence records (Bellis et al. 2020), we obtained subsets of records occurring specifically in fields of sorghum $(n=262)$, millet $(n=157)$, or maize $(n=74)$ according to specimen label data. Although millets include several unrelated species, 46 of 47 herbarium specimens with host identified as "millet" and that also gave the name of a genus indicated Pennisetum spp. rather than Eleusine, Setaria, Digitaria, Panicum, or other less common genera of cultivated "millets". Combined with greater overlap in the distribution of pearl millet growing areas and the range of $S$. hermonthica (National Research Council 1996), our analyses are probably most reflective of parasites on pearl millet.

\section{Specialization predicted from environmental niche models}

To link abiotic environmental variation with host specialization (Q3), we identified areas with optimal conditions for specialization using environmental niche models (ENMs). ENMs are a widely used tool for characterizing suitable habitat for a species based on correlation between environment and observed occurrences of an organism (Elith et al. 2010). An implicit assumption is that organisms should have higher fitness in locations with higher habitat suitability predicted by ENMs (Nagaraju et al. 2013, Wittmann et al. 2016).

We constructed ENMs based on all parasite occurrences (all-occurrence model) and subsets of occurrences associated with a particular host. ENMs were built using MaxEnt (Phillips et al. 2006), a machine learning based method for modeling distributions based on presence-only data. Models were built using $E N$ Meval (Muscarella et al. 2014) to test a variety of tuning parameters. Eight environmental variables were considered, including several associated with differential parasitism on host species (Wilson-Jones 1955, Mohamed et al. 2001), host exudation and production of hormones required for parasite germination (Yoneyama et al. 2013), or parasitism severity (annual rainfall; (Wilson-Jones 1955). Bioclimatic and topographic variables (annual rainfall, mean temperature of the wettest quarter, isothermality, potential evapotranspiration, and topographic wetness index) were obtained from CHELSA (Karger et al. 2017) and ENVIREM (Title and Bemmels 2018) datasets. Soil variables (clay content, nitrogen, and phosphorus) were obtained from SoilGRIDs250m or AfSoilGrids250m (Hengl et al. 2015, 2017). For AfSoilGrids and SoilGrids250m products, we 
downsampled to 1-km resolution using bilinear interpolation with the 'resample' function of the raster package (Hijmans 2020). After downsampling, edaphic variables represent 'average' conditions experienced by a parasite and its close relatives, rather than local heterogeneity associated with a single individual. To characterize the background of the study, we randomly sampled 10,000 points from within a $500 \mathrm{~km}$ radius of all occurrences; the same background points were used to build each of the host-specific models and the all-occurrence model. Habitat suitability values from an ENM of $S$. hermonthica are associated with a large effect resistance allele in sorghum landraces, supporting both the accuracy of the model and some degree of local host adaptation to S. hermonthica (Bellis et al. 2020).

Here, we hypothesized that ENM contrasts, which we define as the difference in habitat suitability between two different ENMs, might also predict variation in performance on different hosts. We created ENM contrasts by subtracting the logistic-transformed ENM output for parasite occurrence on a focal host species from the all host species-occurrence model for each grid cell. Higher potential for specialization on a given host is indicated by more negative values in ENM contrasts (lower bound of -1) and lower potential for specialization is indicated by more positive values (upper bound of 1). Values close to zero can be interpreted as locations where generalist parasites are likely. We calculated the average value within $50 \mathrm{~km}$ of $S$. hermonthica locations from empirical studies as a measure of predicted specialization by ENMs, but similar values were observed at a range of distances (Fig. S3).

We investigated the factors most important for predicting overall occurrence in each model using permutation importance. However, environmental factors most important for determining habitat suitability may differ across space; to identify variables that most influence model prediction at each location, we created limiting factor maps (Elith et al. 2010)with rmaxent (Baumgartner and Wilson in press), fitting models based on the optimal regularization multipliers and feature classes parameters determined by ENMeval (millet: betamultiplier $=3.5$, noproduct, nothreshold; maize: betamultiplier $=0.5$, noproduct, nothreshold, nohinge; sorghum: betamultiplier $=3.5$, nothreshold). After masking to a $200 \mathrm{~km}$ radius of any known $S$. hermonthica occurrence, niche overlap statistics were calculated in ENMTools version 0.2 (Warren et al. 2010), in environmental space with the 'env.overlap' function or in geographic space with the 'calc.niche.overlap' function (Warren et al. 2019).

\section{Linking host distributions, parasite specialization, and parasite distribution}

We used linear mixed effects models (LMMs) to test how parasite specialization in experiments was related to 1) host community variation (relative crop area harvested, Q1), 2) parasite occurrence across hosts (from herbarium records, Q2), and 3) predicted specialization from abiotic environment (host-specific ENM contrasts, Q3). For each of the three predictors of host specialization, we modeled mean relative emergence of an $S$. hermonthica population $i$, on a focal host as follows:

$$
\text { Emergence }_{i j}=b_{0}+b_{1} X_{i}+S_{0 j}+I_{0 i}+R_{0 i}+?_{i j}
$$

where $b_{0}$ and $b_{1}$ are coefficients, $X_{i}$ is the value for one of the predictors of specialization (i.e. crop area harvested, observed parasite herbarium occurrence, or host-specific ENM contrast), $S_{0 j}$ is the random effect of host genotype $j, I_{O i}$ is the random effect of region (Western, Central, or Eastern) for $S$. hermonthica population $i, R_{0 i}$ is the random effect of study (i.e. publication) for $S$. hermonthica population $i$, and ${ }_{i j}$ is the residual error. To assess the significance of our specialization predictors $\left(X_{i}\right)$ as fixed effects, equation 1 was compared to the intercept-only model:

$I_{O i}+R_{O i}+?_{i j}$,

Emergence $_{i j}=b_{0}+S_{0 j}+$

using a Chi-squared test (Table S2). Distribution of host genotypes is likely another factor influencing evolution of host specialization, but data that would allow for inclusion of this effect are currently not available.

Models were built with a Gaussian error distribution using the 'Imer' function from the lme4 package (Bates et al. 2015) and corresponding model summaries and statistical parameters for the fixed effects were cal- 
culated with the lmerTest package using Satterthwaite's method (Kuznetsova et al. 2017). Normality of residual variation was determined by visually inspecting QQplots. Plots were approximately normal and homoscedastic. Models were confirmed to not have significant spatial autocorrelation through calculation of Moran's I of residuals.

\section{Prediction under future climates}

To evaluate potential abiotic driven changes in parasite specialization, we projected ENMs to future climates. We considered Relative Concentration Pathways 4.5 and 8.5, and used climatologies for five CMIP5 models (MPI-ESM-MR, CESM1-BGC, ACCESS-1-0, MIROC5, CMCC-CM) chosen to reduce interdependency (Sanderson et al. 2015). ENMs were projected to 2050 using updated data layers for annual rainfall, mean temperature of the wettest quarter, and isothermality. Soil and ENVIREM variables, for which future projections were not available, remained constant. We calculated the average habitat suitability projected under the five different models for 2050. Considering all pixels within $200 \mathrm{~km}$ of any occurrence record of $S$. hermonthica, we tested whether the habitat suitability score was significantly different in future compared to 1979-2013 using a Wilcoxon signed rank test.

\section{Results:}

Complete host specialization is rare.

We first characterized levels of host specialization observed in $S$. hermonthica populations across Africa. Degree of host specialization was highly variable across the 27 populations studied (Fig. 2 ). Populations were characterized by high emergence on either millet or sorghum only, high emergence on two hosts, or high emergence on all three hosts (Fig. 2). Hierarchical clustering with a cut-point at 0.5 showed five groups: millet specialists (Group 1; $\mathrm{n}=2$ ), millet/sorghum generalists (Group 2; $\mathrm{n}=3$ ), sorghum specialists (Group $3 ; n=7$ ), virulent generalists (Group 4; $n=3$ ), and maize/sorghum specialists (Group $5 ; n=10$; Fig. 2). Besides the 'virulent generalists', all groups included populations from at least two studies, suggesting that differences among studies did not strongly bias clustering. With the exception of millet specialists from Bambey, Senegal, most populations had modest or low levels of specialization as indicated by Pairwise Differentiation Index values (Fig. 2B). These results suggest that although parasites may become locally adapted to commonly cultivated host species, complete specialization is rare.

\section{Distribution of host communities shapes specialization.}

We then investigated whether the distribution of host communities across environments might shape the evolution of host specialization (Q1). S. hermonthica-prone regions covered approximately 628 million hectares, of which $80 \%$ was estimated to have nonzero production of maize, millet, or sorghum. Crop production patterns generally followed rainfall gradients, with greater land area planted to millet in more arid regions of the Sahel, sorghum dominant at lower latitudes and in eastern Sudan, and maize most common in more mesic regions (Fig. 2). In 2000, sorghum or maize were the dominant crop in most $S$. hermonthica-prone areas ( $38 \%$ sorghum; $38 \%$ maize), with $24 \%$ of areas characterized by millet as the major cereal crop.

Specialization observed in experiments was strongly associated with spatial variation in host crop communities (Fig. 3A-C). For parasites on all three hosts, relative host crop area harvested within a $50 \mathrm{~km}$ radius was a strong predictor of mean relative emergence in experimental studies. This finding was especially pronounced for millet $\left(p_{\text {millet }}<0.001 ; \mathrm{b}_{\text {harvest_area }}=1.02 ; \mathrm{LMM}\right.$; Table S2-S3). Relative crop area harvested was also a statistically significant predictor of mean relative emergence on sorghum $\left(p_{\text {sorghum }}=0.04 ; b_{\text {harvest_area }}=0.22\right.$; LMM $)$ and maize $\left(p_{\text {maize }}=0.04 ; b_{\text {harvest_area }}=0.23\right.$; LMM; Table S2-S3). These results are consistent with the conclusion that parasites adapt to the most abundant host in a particular region and also suggest that relative parasite emergence is a reasonable proxy for fitness (Fig. 1).

Host specialization may structure parasite distributions. 
Our analyses provided little support for a positive relationship between specialization and parasite occurrence on different hosts (Q2; Fig. 3D-F). Within a 50-km radius of all 27 locations with parasite emergence data, 15 locations had at least one parasite occurrence record with information about host of origin. Proportion of records on a particular host for these 15 Striga populations was not a statistically significant predictor of emergence on maize $\left(p_{\text {maize }}=0.07 ; \mathrm{b}_{\text {occurrence }}=0.18 ; \mathrm{LMM}\right)$, millet $\left(p_{\text {millet }}=0.06 ; \mathrm{b}_{\text {occurrence }}=0.21 ; \mathrm{LMM}\right)$, or sorghum $\left(p_{\text {sorghum }}=0.57 ; \mathrm{b}_{\text {occurrence }}=0.05 ; \mathrm{LMM}\right.$; Table S2). Availability of additional parasite occurrence and performance data could reveal a stronger relationship between specialization and patterns of parasite occurrence on different hosts.

Parasite abiotic niche reflects host environmental tolerance.

To characterize the abiotic basis of patterns of host specialization, we built environmental niche models (ENMs) for potentially host-specialized parasite populations by excluding all occurrences with unknown host or with a non-focal host (Fig. S2). Despite a smaller number of observations compared to the alloccurrence model, ENMs had good predictive accuracy for each of the host-specific models. Area under the receiver operating characteristic curve (AUC) for the test set was 0.848 for the all-occurrence model $(n$ $=1049)$ compared to 0.850 for the sorghum-only model $(n=262), 0.908$ for the millet-only model $(n=$ $157)$, and 0.841 for the maize-only model $(n=74)$.

Modeled niches generally reflected known environmental tolerances of hosts, with millet-specific models predicting high habitat suitability in low nitrogen $(\mathrm{N})$, low rainfall environments but maize-specific models predicting higher habitat suitability in environments characterized by more nitrogen-rich soils and higher rainfall (Fig. 4A-D). Across all host species, annual rainfall and soil $\mathrm{N}$ were generally among the most important predictors of parasite occurrence (Table S4). Annual rainfall was the most common factor limiting habitat suitability for maize-parasitizing $S$. hermonthica ( $59 \%$ of all grid cells; $10^{\text {th }}-90^{\text {th }}$ percentile for grid cells with habitat suitability [?] 0.5: $854-1537 \mathrm{~mm} / \mathrm{yr}$ ) but was also strongly limiting for parasites of sorghum ( $41 \%$ of cells; $10^{\text {th }}-90^{\text {th }}$ percentile: $509-1197 \mathrm{~mm} / \mathrm{yr}$ ) and millet (32\% of cells; $10^{\text {th }}-90^{\text {th }}$ percentile: $441-1164$ $\mathrm{mm} / \mathrm{yr}$ ) (Fig. S4). Mean temperature of the wettest quarter was also an important predictor of S. hermonthica parasitizing millet (Table S4) and frequently limited habitat suitability for millet-parasitizing populations (Fig. S4). Soil clay content was an important predictor for S. hermonthica occurrence on sorghum (Table S4), but was only limiting for millet-parasitizing populations in eastern Sudan and sorghum-parasitizing populations in western Senegal (Fig. S4). Higher soil clay content has been anecdotally associated with parasitism on sorghum in general (Mohamed et al. 2001) and in Sudan (Wilson-Jones 1955).

In agricultural as well as natural ecosystems, more productive environments may be associated with increased availability of alternate hosts, favoring generalists (Thrall et al. 2007). Parasitism on maize was associated with increased environmental quality, with habitat suitability peaking in locations of higher crop productivity (Fig. 4E). Combined crop yield per area harvested was significantly greater where $S$. hermonthica parasitizes maize compared to millet ( $p<0.001$, Wilcoxon rank sum test) but not compared to sorghum $(p=0.2)$, consistent with greater niche overlap between maize and sorghum (Table S5). We observed a weak trend towards reduced parasite specialization with increasing environmental productivity (Fig. $4 \mathrm{~F} ; p=0.15$, Chisquare goodness-of-fit test for linear regression model). Taken together, parasite ENMs are highly sensitive to differences in multivariate environmental tolerance of hosts, consistent with host cultivation on a gradient from marginal, warm environments (pearl millet) to more productive, cooler environments (maize).

\section{Abiotic gradients shape distribution of millet and sorghum specialists.}

ENM contrasts show where host niches differ most in multivariate environmental space. If abiotic environment is associated with host specialization, specialists may be most likely where environmental axes most strongly differentiate hosts in niche space (Futuyma and Moreno 1988). Consistent with this idea, ENM contrasts were strongly predictive of variation in $S$. hermonthica performance on host species for which specialist parasite populations have been previously reported (Fig. 3G-H). ENM contrasts were most strongly associated with mean relative emergence on pearl millet $\left(p_{\text {millet }}<0.001 ; b_{\mathrm{ENM}}=-0.68 ; \mathrm{LMM}\right)$ and were also significantly associated with mean relative emergence on sorghum $\left(p_{\text {sorghum }}=0.0067 ; b_{\mathrm{ENM}}=-0.50 ; \mathrm{LMM}\right)$ 
(Table S2, S6). In contrast, host specialization predicted by ENM contrasts was not strongly associated with mean relative emergence on maize $\left(p_{\text {maize }}=0.19, \mathrm{~b}_{\mathrm{ENM}}=0.15\right.$; LMM; Table S2, S6).

Future change in parasite distributions

To investigate potential changes in host-specialization over time, we projected ENMs to future climates (Fig. S5). By 2050, we predicted an overall increase in habitat suitability for S. hermonthica, with median increase in habitat suitability in its current range of 0.07 under RCP $4.5(p<0.001$; Wilcoxon signed rank test comparing habitat suitability of grid cells in present vs. 2050) and 0.09 under RCP $8.5(p<0.001$; Wilcoxon signed rank test; Table S7). Habitat suitability increased most for millet-parasitizing populations, followed by maize-parasitizing $S$. hermonthica (Table S7). Changes in habitat suitability were heterogenous across space, with many regions in the Sudano-Sahelian zone of west Africa becoming less suitable for $S$. hermonthica and its hosts (Sultan et al. 2013) but central and east Africa generally becoming more suitable (Fig. S5).

\section{Discussion:}

An outstanding goal in evolutionary ecology is understanding how reciprocal host and parasite interactions shape distribution of species. For multi-host parasites, parasite eco-evolutionary dynamics depend on many components including environmental tolerance, host compatibility, genetic diversity, and source-sink dynamics (Woolhouse et al. 2001, Dobson 2004, Gandon 2004, Bellis et al. 2020). Although parasite abundance is often constrained by local environment (Wu et al. 2019), it is rarely considered how parasite evolution may also be indirectly affected by host abiotic environmental tolerance. Here we present a framework to understand broad-scale interactions between $S$. hermonthica and its common hosts across abiotic gradients. Combining host distributional and empirical data from diverse populations of a parasitic plant, we find that regional abundance of a particular host is associated with local specialization on that crop by parasites. By controlling host distributions, abiotic gradients may indirectly shape patterns of parasite local adaptation to host communities and consequently, distributions of generalist and specialist parasites.

Host communities in natural ecosystems can be highly heterogeneous, impacting parasite evolution. In theory, low host heterogeneity favors parasite specialization, whereas high host heterogeneity favors generalism (Futuyma and Moreno 1988). However, strong empirical support for this phenomenon comes from relatively few systems (Legros and Koella 2010, Fecchio et al. 2018, Gibson et al. 2020) and even fewer at continent scale. With well-characterized host community distributions (Monfreda et al. 2008), and host cultivation patterns strongly tied to abiotic gradients (Fig. 2), S. hermonthica parasitic plants are a valuable system for developing a predictive framework of host specialization. Unlike well-studied microbial parasites with short generation times (Penczykowski et al. 2016), S. hermonthica may be able to maintain long-term adaptive potential due to a long-lived seedbank (>10 yrs; (Bebawi et al. 1984) and substantial genetic diversity from its outcrossing breeding system (Bozkurt et al. 2015, Unachukwu et al. 2017). Whereas repeated selection could exhaust parasite genetic diversity under limited migration and mutation, longer parasite generation times relative to hosts (driven by a long-lived seedbank) help maintain parasite diversity and promote adaptation to changing environments (Gandon and Michalakis 2002).

Our results suggest patterns of host cultivation are strongly associated with parasite adaptation to local host communities (Q1; Fig. 3A-C). Using crop harvest data to approximate relative host abundance (Fig. 2), we found that $S$. hermonthica emergence was highest on the host species most commonly grown at location of origin of parasite populations (Table S3). This pattern satisfies the 'home vs. away' criterion described by Kawecki \& Ebert (2004), as mean fitness of the parasite is highest in its 'home' host environment compared to 'away' hosts. Although 'home vs. away' patterns sometimes conflict with 'foreign vs. local' genotype contrasts when one environment is relatively higher quality, we did not see evidence for such a conflict. Instead different parasite populations performed best on different hosts. Higher fitness on 'home' hosts was apparent despite the fact that we were not able to find high resolution crop distributional data prior to the year 2000, whereas empirical studies were based on S. hermonthica populations from field and pot trials that were sampled or tested 12-25 years earlier. This may be because host community composition (relative area 
planted to each host crop) remained roughly constant in East and West Africa during this period, although total area harvested increased dramatically (Fig. S1). Evidence supporting a link between specialization and patterns of parasite occurrence on different hosts (Q2) was weaker, likely due to the small number of parasite records with host annotations that were also near the origin of $S$. hermonthica populations tested in experiments (Fig. 3D-F).

If parasite populations adapt to local host communities, it follows that the abiotic environments which shape host communities also shape distributions of generalist and specialist parasites (Q3). In support of our ENM-based approach, we found that host-specific ENMs readily distinguished parasite populations along environmental axes associated with differences in host performance (Fig. 4A-D). These axes may correspond to a multivariate 'optimum' that differs for parasites on different host species, possibly due to host environmental tolerances (Reynolds et al. 2015). Parasites may perform best on hosts that are stressed enough to exude chemical signals for recruitment of mycorrhizae, signals which are also required for parasites to sense hosts and germinate (Yoneyama et al. 2010). However, parasite reproductive fitness may be low if hosts are too stressed to provide adequate nutrition. Supporting an important role of abiotic environment in shaping distribution of specialist parasites, we found significant associations between ENM contrasts and relative parasite emergence on pearl millet and sorghum observed in common garden experiments and in pot trials (Fig. 3G-H; Table S6).

We did not, however, observe an association between ENM contrasts and mean relative emergence for parasites of maize (Fig. 3I). Given that relative crop area harvested did predict emergence on maize (Fig. 3C; Table S2), it may be that ENM contrasts perform poorly if alternate hosts are common. Alternate hosts may be particularly common in areas with the highest quality environments, which are also those predicted to have highest habitat suitability for parasites of maize (Fig. 4). For example, ENM contrasts predicted high suitability for maize specialization in central Nigeria (Fig. 5). However, sorghum was the dominant crop in this area in 2000 and other hosts are commonly grown (Fig. S1); indeed, most tested parasite populations from Nigeria appear to be maize/sorghum generalists (Fig. 2). In addition to increased diversity of hosts in higher quality environments (Thrall et al. 2007), the observed lack of parasite specialization on maize could result from coevolutionary history. Both sorghum and pearl millet originated in Africa, with evidence for centers of domestication for sorghum in eastern Sudan (Winchell et al. 2017) and for pearl millet in the western Sahara (Burgarella et al. 2018). In contrast, maize was domesticated in Mexico about 9,000 years ago (Matsuoka et al. 2002, Piperno et al. 2009) and improved varieties bred specifically for African environments were not available until the 1980s and 1990s (Evenson and Gollin 2003).

Consequently, parasite populations in published experiments may not yet have reached a local fitness optimum with respect to maize virulence traits. For example, low or no emergence was recorded on maize for $S$. hermonthica from two locations (Abu Naama, Sudan and Samuru, Nigeria), although both locations had high predicted habitat suitability for parasitism on maize (Fig. S2). However, in the year of each respective trial, only $2 \%$ (Sudan, 1979) or 12\% (Nigeria, 1975) of total crop area harvested of maize, millet, and sorghum was planted to maize (Food and Agriculture Organization of the United Nations 2020). Thirteen years later, when relative area of maize harvested in Nigeria had more than doubled (Fig. S1), Kim et al. (1994) observed higher emergence on maize than either sorghum or pearl millet in 10 of 12 locations. Kim et al. (1994) also described poor reproductive success (post emergence) of parasites on maize relative to sorghum. If contemporary S. hermonthica populations are better adapted to maize, for example through evolution of reduced virulence but increased seed set, ENM contrasts might show stronger associations with emergence.

Taken together, our study suggests that abiotic environmental gradients shape parasite adaptation to cultivated host communities across continental Africa. Our results indicate that parasites specialize on abundant hosts in environments that strongly distinguish hosts in multivariate niche space or generalize where diverse hosts are available. If underlying abiotic drivers can predict host specialization, one promising application is the potential to inform management efforts. For example, if pearl millet production increases in the Guinean region of West Africa (Fig. S5), shifting patterns in host cultivation could provide a 'window of opportunity' 
for intensive management before parasite populations become locally adapted to host species or genotypes new to a region. Complete prevention of parasite reproduction on new hosts, through hand-weeding or other means, would be critical to take advantage of any 'lag' in population dynamics. The modeling framework developed here could be further extended to tailor management strategies for S. hermonthica and other parasites under shifting abiotic gradients linked to climate change.

\section{Acknowledgments:}

We thank the many collectors, volunteers, and herbarium curators who made the niche modeling work possible and are particularly grateful to Andrew Budden (Royal Botanic Gardens Kew) and Germinal Rouhan (Muséum National d'Histoire Naturelle, Paris). This study is based on work supported by an NSF Postdoctoral Research Fellowship in Biology to E.S.B. under Grant 1711950.

\section{References:}

Bates, D. et al. 2015. Fitting linear mixed-effects models using lme4. - Journal of Statistical Software in press.

Baumgartner, J. and Wilson, P. \{rmaxent\}: Tools for working with Maxent in R. in press.

Bebawi, F. F. et al. 1984. Longevity of Witchweed (Striga asiatica) Seed. - Weed Science 32: 494-497.

Bebawi, F. F. et al. 1986. Germination, Host Preference, and Phenolic Content of Witchweed (Striga hermonthica) Seed Populations. - Weed Science 34: 529-532.

Bellis, E. S. et al. 2020. Genomics of sorghum local adaptation to a parasitic plant. - Proceedings of the National Academy of Sciences of the United States of America 117: 4243-4251.

Bozkurt, M. L. et al. 2015. Genetic diversity of East and West African Striga hermonthica populations and virulence effects on a contrasting set of sorghum cultivars. - Weed Research 55: 71-81.

Brown, J. H. 1984. On the Relationship between Abundance and Distribution of Species. - The American Naturalist 124: 255-279.

Brown, J. S. and Pavlovic, N. B. 1992. Evolution in heterogeneous environments: Effects of migration on habitat specialization. - Evolutionary Ecology 6: 360-382.

Buckley, L. B. and Roughgarden, J. 2005. Effect of species interactions on landscape abundance patterns. Journal of Animal Ecology 74: 1182-1194.

Burgarella, C. et al. 2018. A western Sahara centre of domestication inferred from pearl millet genomes. Nature Ecology and Evolution 2: 1377-1380.

Clarke, C. R. et al. 2019. Molecular Dialog Between Parasitic Plants and Their Hosts. - Annu. Rev. Phytopathol. 57: 279-299.

Clausen, J. et al. 1940. Experimental studies on the nature of species I. Effects of varied environments on western North American plants. Carnegie Institution of Washington. - Carnegie Institution of Washington.

Cook, C. E. et al. 1966. Germination of Witchweed (Striga lutea Lour.): Isolation and Properties of a Potent Stimulant. - Science 154: 1189.

Cui, S. et al. 2018. Host lignin composition affects haustorium induction in the parasitic plants Phtheirospermum japonicum and Striga hermonthica. - New Phytologist 218: 710-723.

Devictor, V. et al. 2010. Defining and measuring ecological specialization. - Journal of Applied Ecology 47: $15-25$.

Dobson, A. 2004. Population dynamics of pathogens with multiple host species. - American Naturalist in press. 
Elith, J. et al. 2010. The art of modelling range-shifting species. - Methods in Ecology and Evolution 1: $330-342$.

Evenson, R. E. and Gollin, D. 2003. Assessing the impact of the Green Revolution, 1960 to 2000. - Science 300: $758-762$.

Fecchio, A. et al. 2018. Host community similarity and geography shape the diversity and distribution of haemosporidian parasites in Amazonian birds. - Ecography 41: 505-515.

Fecchio, A. et al. 2019. Climate variation influences host specificity in avian malaria parasites. - Ecology Letters 22: $547-557$.

Food and Agriculture Organization of the United Nations 2020. FAOSTAT Database. - Rome, Itay: FAO

Futuyma, D. J. and Moreno, G. 1988. The evolution of ecological specialization. - Annual review of ecology and systematics. Vol. 19: 207-233.

Gandon, S. 2004. Evolution of multihost parasites. - Evolution 58: 455-469.

Gandon, S. and Michalakis, Y. 2002. Local adaptation, evolutionary potential and host-parasite coevolution: Interactions between migration, mutation, population size and generation time. - Journal of Evolutionary Biology 15: 451-462.

Gibson, A. K. et al. 2020. The evolution of parasite host range in heterogeneous host populations. - Journal of Evolutionary Biology 33: 773-782.

Hengl, T. et al. 2015. Mapping soil properties of Africa at $250 \mathrm{~m}$ resolution: Random forests significantly improve current predictions. - PLoS ONE 10: 1-26.

Hengl, T. et al. 2017. SoilGrids250m: Global gridded soil information based on machine learning. - PLoS ONE 12: 1-40.

Hereford, J. 2009. A quantitative survey of local adaptation and fitness trade-offs. - American Naturalist 173: $579-588$.

Hijmans, R. J. 2020. raster: Geographic Data Analysis and Modeling. in press.

Holt, R. D. 2020. Reflections on niches and numbers. - Ecography 43: 387-390.

Honaas, L. A. et al. 2013. Functional genomics of a generalist parasitic plant: Laser microdissection of host-parasite interface reveals host-specific patterns of parasite gene expression. - BMC Plant Biology in press.

Johnson, N. R. et al. 2019. Compensatory sequence variation between trans-species small RNAs and their target sites. - eLife 8: 1-17.

Kaltz, O. and Shykoff, J. A. 1998. Local adaptation in host-parasite systems. - Heredity 81: 361-370.

Karger, D. N. et al. 2017. Climatologies at high resolution for the earth's land surface areas. - Scientific Data 4: $1-20$.

Kawecki, T. J. and Ebert, D. 2004. Conceptual issues in local adaptation. - Ecology Letters 7: 1225-1241.

Kim, S. K. et al. 1994. Responses of maize, sorghum and millet host plants to infestation by Striga hermonthica. - Crop Protection 13: 582-590.

King, S. B. and Zummo, N. 1977. Physiologic specialization in Striga hermonthica in West Africa. - Plant Disease Reporter 61: 770-773.

Koskella, B. 2014. Bacteria-Phage Interactions across Time and Space: Merging Local Adaptation and TimeShift Experiments to Understand Phage Evolution. - The American Naturalist 184: S9-S21. 
Koskella, B. et al. 2011. Local biotic environment shapes the spatial scale of bacteriophage adaptation to bacteria. - American Naturalist 177: 440-451.

Krasnov, B. R. et al. 2004. Relationships between parasite abundance and the taxonomic distance among a parasite's host species: An example with fleas parasitic on small mammals. - International Journal for Parasitology 34: 1289-1297.

Kuznetsova, A. et al. 2017. lmerTest Package: Tests in Linear Mixed Effects Models . - Journal of Statistical Software in press.

Laine, A. L. 2005. Spatial scale of local adaptation in a plant-pathogen metapopulation. - Journal of Evolutionary Biology 18: 930-938.

Legros, M. and Koella, J. C. 2010. Experimental evolution of specialization by a microsporidian parasite. BMC Evolutionary Biology 10: 1-8.

Leimu, R. and Fischer, M. 2008. A Meta-Analysis of Local Adaptation in Plants. - PLOS ONE 3: e4010.

Lopez, L. et al. 2019. Transcriptomics of host-specific interactions in natural populations of the parasitic plant purple witchweed (Striga hermonthica). - Weed Science 67: 397-411.

Matsuoka, Y. et al. 2002. A single domestication for maize shown by multilocus microsatellite genotyping. Proceedings of the National Academy of Sciences of the United States of America 99: 6080-6084.

Mohamed, K. et al. 2001. The Genus $<\mathrm{i}>$ Striga $<\mathrm{i}>$ (Scrophulariaceae) in Africa. - Annals of the Missouri Botanical Garden 88: 60-103.

Monfreda, C. et al. 2008. Farming the planet: 2. Geographic distribution of crop areas, yields, physiological types, and net primary production in the year 2000. - Global Biogeochemical Cycles 22: 1-19.

Muscarella, R. et al. 2014. ENMeval: An R package for conducting spatially independent evaluations and estimating optimal model complexity for Maxent ecological niche models . - Methods in Ecology and Evolution 5: 1198-1205.

Nagaraju, S. K. et al. 2013. Do ecological niche model predictions reflect the adaptive landscape of species?: A test using Myristica malabarica Lam., an endemic tree in the Western Ghats, India. - PLoS ONE 8: 1-13.

National Research Council 1996. Lost Crops of Africa: Volume I: Grains. - The National Academies Press.

Osorio-Olvera, L. et al. 2019. On population abundance and niche structure. - Ecography 42: 1415-1425.

Parker, C. and Reid, D. 1979. Host specificity in Striga species: Some preliminary observations. - Proceedings of the Second International Symposium on Parasitic Weeds: 79-90.

Penczykowski, R. M. et al. 2016. Understanding the ecology and evolution of host-parasite interactions across scales. - Evolutionary Applications 9: 37-52.

Peterson, M. L. et al. 2019. Incorporating local adaptation into forecasts of species' distribution and abundance under climate change. - Global Change Biology 25: 775-793.

Phillips, S. J. et al. 2006. Maximum entropy modeling of species geographic distributions. - Ecological Modelling 190: 231-259.

Piperno, D. R. et al. 2009. Starch grain and phytolith evidence for early ninth millennium B.P. maize from the Central Balsas River Valley, Mexico. - Proceedings of the National Academy of Sciences of the United States of America 106: 5019-5024.

Poisot, T. et al. 2012. A comparative study of ecological specialization estimators. - Methods in Ecology and Evolution 3: 537-544. 
Poulin, R. 2005. Relative infection levels and taxonomic distances among the host species used by a parasite: Insights into parasite specialization. - Parasitology 130: 109-115.

Poulin, R. et al. 2012. Scaling up from epidemiology to biogeography: local infection patterns predict geographical distribution in fish parasites. - Journal of Biogeography 39: 1157-1166.

Pulliam, H. R. 1988. Sources, Sinks, and Population Regulation. - The American Naturalist 132: 652-661.

R Core Team 2020. R: A language and environment for statistical computing. in press.

Ramaiah, K. V. 1983. Striga Research at ICRISAT Upper Volta Center. - Proceedings of the Second International Workshop on Striga.: 53-60.

Reynolds, T. W. et al. 2015. Environmental impacts and constraints associated with the production of major food crops in Sub-Saharan Africa and South Asia. - Food Security 7: 795-822.

Rich, P. J. and Ejeta, G. 2008. Towards effective resistance to Striga in African maize. - Plant Signaling and Behavior 3: 618-621.

Rodenburg, J. et al. 2016. Parasitic weed incidence and related economic losses in rice in Africa. - Agriculture, Ecosystems \& Environment 235: 306-317.

Runo, S. and Kuria, E. K. 2018. Habits of a highly successful cereal killer, Striga. - PLOS Pathogens 14: e1006731.

Sanderson, B. M. et al. 2015. Addressing interdependency in a multimodel ensemble by interpolation of model properties. - Journal of Climate 28: 5150-5170.

Shahid, S. et al. 2018. MicroRNAs from the parasitic plant Cuscuta campestris target host messenger RNAs. - Nature 553: 82-85.

Slatkin, M. 1973. Gene flow and selection in a cline. - Genetics 75: 733-756.

Stephens, P. R. et al. 2016. The macroecology of infectious diseases: a new perspective on global-scale drivers of pathogen distributions and impacts. - Ecology letters 19: 1159-1171.

Sultan, B. et al. 2013. Assessing climate change impacts on sorghum and millet yields in the Sudanian and Sahelian savannas of West Africa. - Environmental Research Letters in press.

Tack, A. J. M. et al. 2014. The impact of spatial scale and habitat configuration on patterns of trait variation and local adaptation in a wild plant parasite. - Evolution 68: 176-189.

Thrall, P. H. and Burdon, J. J. 1997. Host-Pathogen Dynamics in a Metapopulation Context: The Ecological and Evolutionary Consequences of Being Spatial Source. - Journal of Ecology 85: 743-753.

Thrall, P. H. et al. 2002. Local adaptation in the Linum marginale-Melampsora lini host-pathogen interaction. - Evolution 56: 1340-1351.

Thrall, P. H. et al. 2007. Coevolution of symbiotic mutualists and parasites in a community context. - Trends in Ecology and Evolution 22: 120-126.

Timko, M. P. et al. 2012. Host Resistance and Parasite Virulence in Striga-Host Plant Interactions: A Shifting Balance of Power. - Weed Science 60: 307-315.

Title, P. O. and Bemmels, J. B. 2018. ENVIREM: an expanded set of bioclimatic and topographic variables increases flexibility and improves performance of ecological niche modeling. - Ecography 41: 291-307.

Unachukwu, N. N. et al. 2017. Genetic diversity and population structure of Striga hermonthica populations from Kenya and Nigeria. - Weed Research 57: 293-302.

Warren, D. L. et al. 2010. ENMTools: A toolbox for comparative studies of environmental niche models. Ecography 33: 607-611. 
Warren, D. L. et al. 2019. New methods for measuring ENM breadth and overlap in environmental space. Ecography 42: 444-446.

Wells, K. and Clark, N. J. 2019. Host Specificity in Variable Environments. - Trends in Parasitology 35: $452-465$.

Wells, K. et al. 2019. Global patterns in helminth host specificity: phylogenetic and functional diversity of regional host species pools matter. - Ecography 42: 416-427.

Wilson-Jones, K. 1955. Further experiments on witchweed control. II. The existence of physiological strains of Striga hermonthica. - Empire Journal of Experimental Agriculture 23: 206-213.

Winchell, F. et al. 2017. Evidence for Sorghum Domestication in Fourth Millennium BC Eastern Sudan: Spikelet Morphology from Ceramic Impressions of the Butana Group. - Current Anthropology 58: 673-683.

Wittmann, M. E. et al. 2016. Confronting species distribution model predictions with species functional traits. - Ecology and Evolution 6: 873-879.

Woolhouse, M. E. J. et al. 2001. Population biology of multihost pathogens. - Science 292: 1109-1112.

$\mathrm{Wu}, \mathrm{Q}$. et al. 2019. Environmental variation mediates the prevalence and co-occurrence of parasites in the common lizard, Zootoca vivipara. - BMC Ecology 19: 1-11.

Yoneyama, K. et al. 2010. Strigolactones as germination stimulants for root parasitic plants. - Plant \& cell physiology 51: 1095-1103.

Yoneyama, K. et al. 2013. Nitrogen and phosphorus fertilization negatively affects strigolactone production and exudation in sorghum. - Planta 238: 885-894.

\section{Figure Legends}

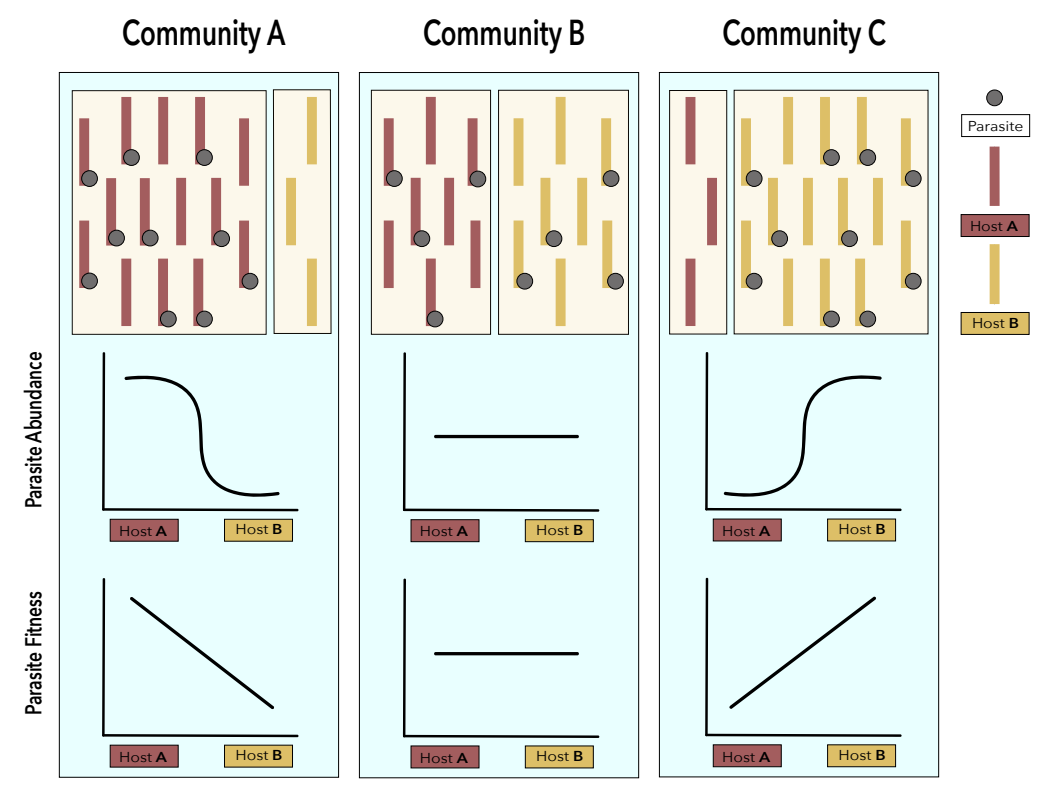

Fig. 1. Parasite adaptation to local host community. Predictions are shown for three scenarios. Specialist 
parasites (characterized by higher fitness on the most abundant host) are selected for in communities A and $\mathrm{C}$, and generalist parasites (with similar fitness on diverse hosts) are selected for in community B. Parasite abundance may decrease more rapidly with decreasing prevalence of alternate hosts due to processes such as dispersal limitation.

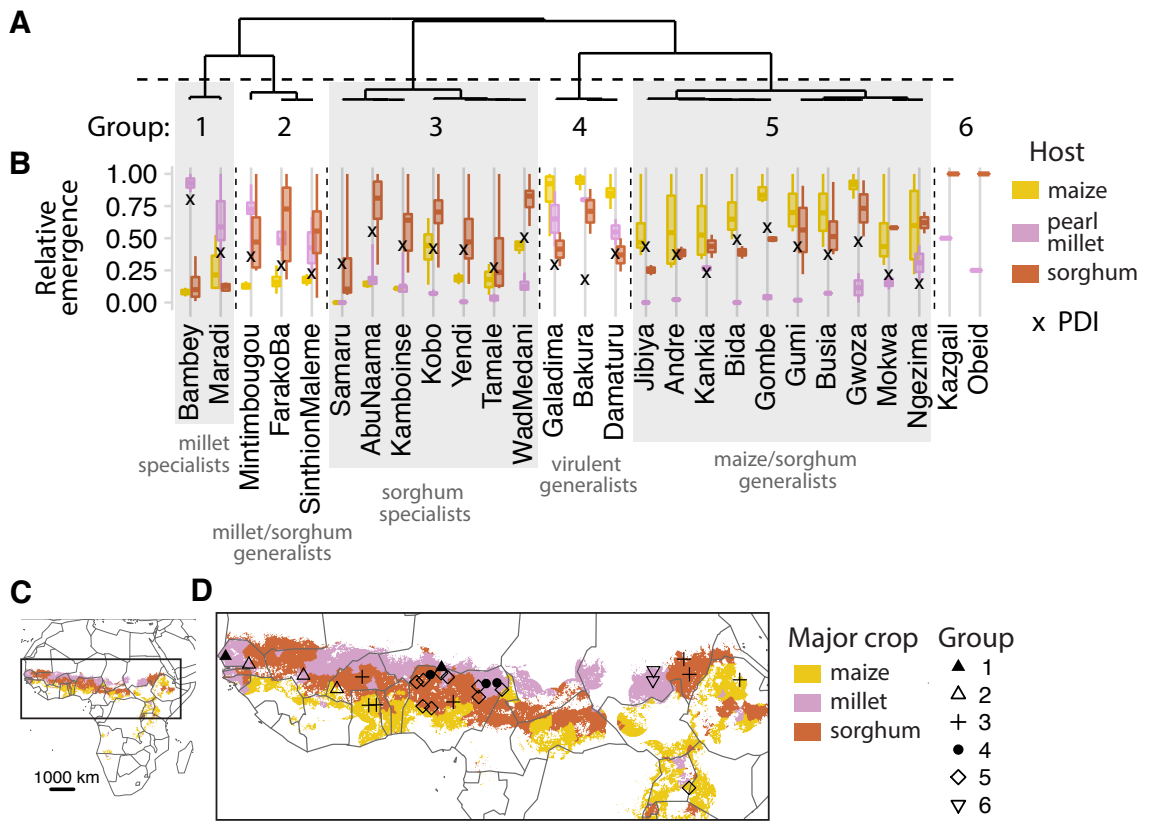

Fig. 2. Distribution of generalist and specialist $S$. hermonthica in published studies. Relative emergence was calculated as the mean number of parasites emerged on a particular host species, relative to the highest emergence observed for any tested host genotype. A) Dendrogram obtained from hierarchical clustering of mean relative emergence values for three hosts. B) Relative emergence on maize, sorghum, and pearl millet for S. hermonthica from 27 localities. Boxplots represent the distribution of relative emergence values across all tested genotypes of a host species. Whiskers extend to the minimum and maximum values and boxes indicate the first and third quartiles. The black ' $x$ ' shows the value of the Paired Difference Index (PDI) calculated for each population, where values closer to 1 indicate more specialized populations. Vertical dashed lines demarcate $S$. hermonthica clusters determined in A. Parasites were classified in the following groups: 1) millet specialists, 2) millet/sorghum generalists, 3) sorghum specialists, 4) virulent generalists (where high emergence is observed across multiple host species), 5) maize/sorghum generalists, and 6) two populations that were excluded from the clustering analysis because emergence on maize was not tested. C) Host crop production in $S$. hermonthica-prone regions. Colored regions indicate the most abundant host crop based on estimated area harvested in 2000. Note pearl millet is not distinguished from other millets in harvest area data. D) Inset map. Black symbols $(n=27)$ indicate group assignments from (A) and the location of origin of $S$. hermonthica populations tested for emergence in empirical studies. 


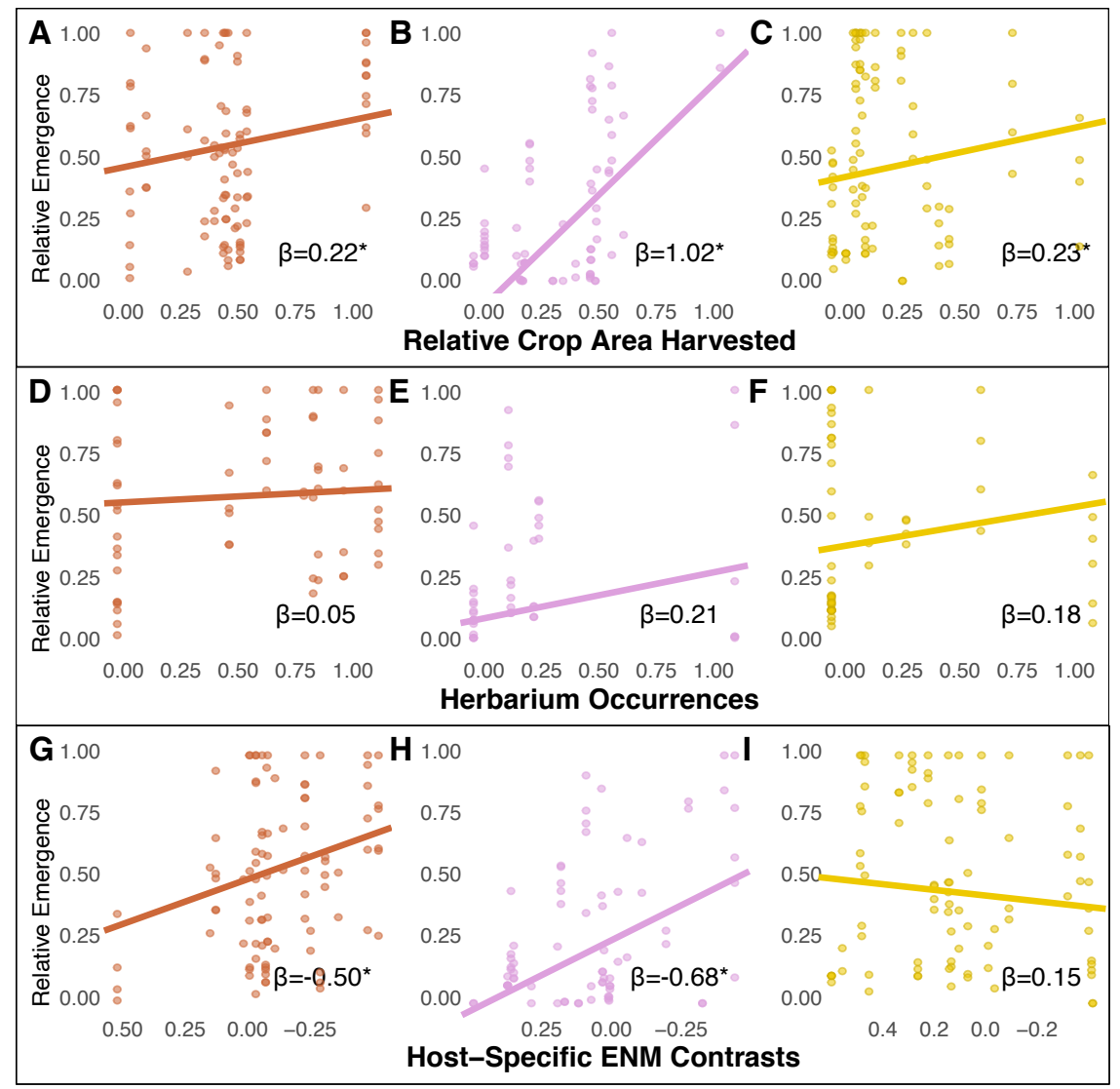

Host: maize pearl millet sorghum

Fig. 3. The relationship between mean relative emergence from empirical studies and metrics of predicted specialization for focal hosts. Each point is representative of the relative emergence of a parasite population on a single host genotype and predicted specialization based on either A-C) relative crop harvested area, DF) herbarium occurrences, or G-I) host-specific ENM contrasts and plotted against actualized specialization (mean relative emergence). Lines are shown based on coefficient estimates from linear mixed models. Beta coefficients with an asterisk indicate that the added fixed effect of a specialization parameter is significant for model predictions of mean relative emergence. The axis for host-specific ENM contrasts (G-I) is inverted such that more negative ENMs reflect higher specialization (see Materials and Methods) 

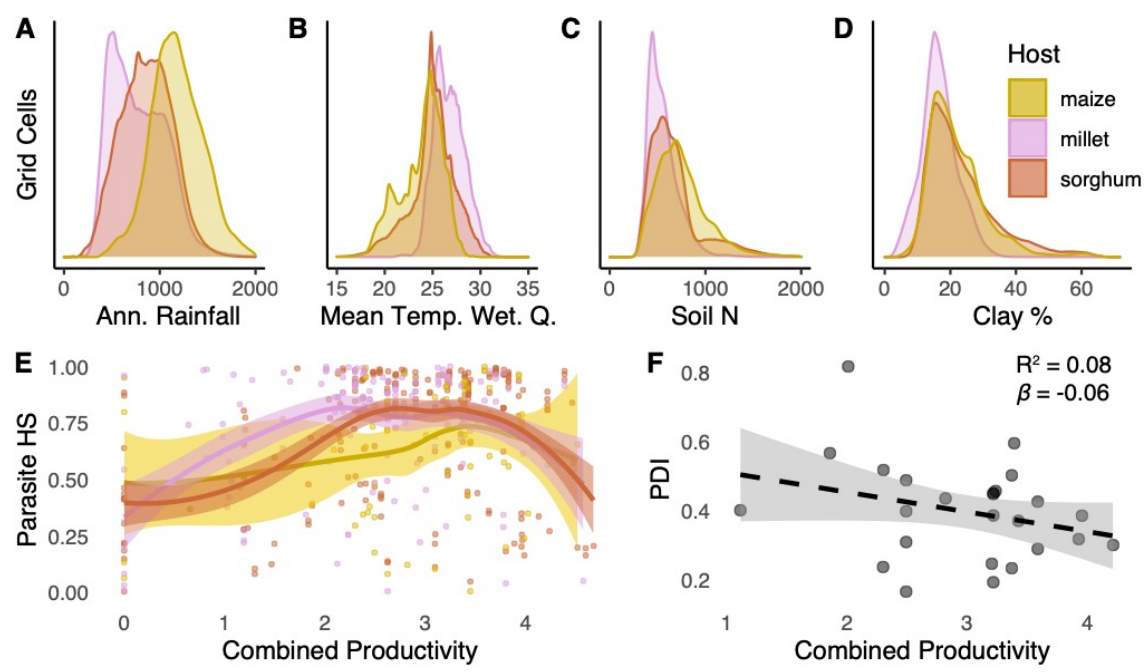

Fig. 4. Parasite ENMs reflect host abiotic tolerances. A-D) Distribution of environmental values for grid cells in core habitat (habitat suitability [?] 0.5) for ENMs based on subsets on occurrence records on different hosts. E) Habitat suitability (HS) as a function of combined host productivity (yield per hectare) at locations of maize-, millet-, and sorghum-parasitizing S. hermonthica from natural history observations. Curves were fit using local polynomial regression. HS peaks in locations of higher productivity for parasites of maize compared to millet or sorghum. F) Host specialization measured as the Paired Difference Index (PDI) for $n=25 \mathrm{~S}$. hermonthicapopulations tested for emergence in previous studies. Shaded areas in (E) and (F) indicate 90\% confidence intervals. Ann. Rainfall: annual rainfall (mm/yr); Mean Temp. Wet. Q.: mean temperature of the wettest quarter $\left({ }^{\circ} \mathrm{C}\right)$; soil $\mathrm{N}$ : soil nitrogen $(\mathrm{g} / \mathrm{kg})$; clay: clay fraction (weight \%)

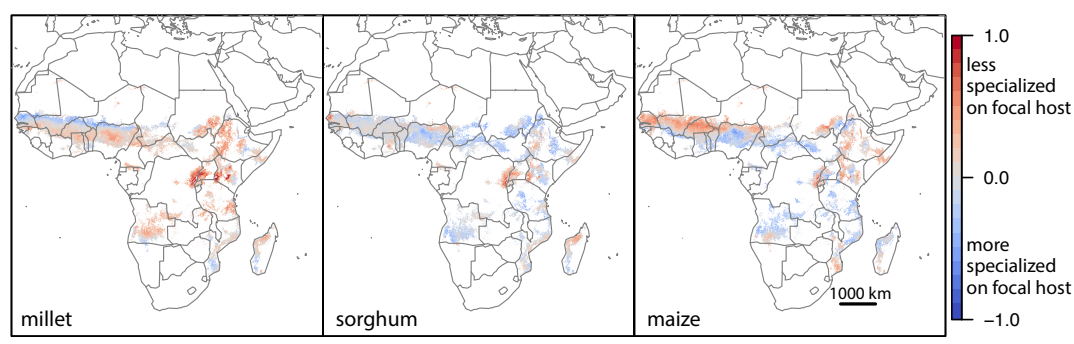

Figure 5. ENM contrasts to predict host specialization by $S$. hermonthica . Logistic output from each of the host-specific models was subtracted from the all-occurrence model, so that values close to 0 indicate similar predicted habitat suitability, negative values indicate higher habitat suitability in the host-specific model, and positive values indicate higher habitat suitability in the all-occurrence model. Pixels with habitat suitability in the all-occurrence model $<0.2$ are not shown. 